\title{
Effects of afferent input on the breathing pattern continuum in the tambaqui (Colossoma macropomum)
}

\author{
Stephen G. Reid ${ }^{\mathrm{a}, *}$, Lena Sundin ${ }^{1}$, Luis Henrique Florindo ${ }^{\mathrm{b}}$, Francisco \\ Tadeu Rantin ${ }^{\mathrm{b}}$, William K. Milsom ${ }^{\mathrm{c}}$ \\ a Department of Life Sciences, University of Toronto at Scarborough, 1265 Military Trail, Toronto, Ont., Canada M1C 1 A4 \\ ${ }^{\mathrm{b}}$ Department of Physiological Sciences, Federal University of São Carlos, 13565-905 São Carlos, SP, Brazil \\ ${ }^{\mathrm{c}}$ Department of Zoology, University of British Columbia, Vancouver, BC, Canada V6T 1 Z4
}

Accepted 18 February 2003

\begin{abstract}
This study used a decerebrate and artificially-ventilated preparation to examine the roles of various afferent inputs in breathing pattern formation in the tambaqui (Colossoma macropomum). Three general breathing patterns were observed: (1) regular breathing; (2) frequency cycling and (3) episodic breathing. Under normoxic, normocapnic conditions, $50 \%$ of control fish exhibited regular continuous breathing and 50\% exhibited frequency cycling. Denervation of the gills and oro-branchial cavity promoted frequency cycling. Central denervation of the glossopharyngeal and vagus nerves produced episodic breathing. Regardless of the denervation state, hyperoxia produced either frequency cycling or episodic breathing while hypoxia and hypercarbia shifted the pattern to frequency cycling and continuous breathing. We suggest that these breathing patterns represent a continuum from continuous to episodic breathing with waxing and waning occupying an intermediate stage. The data further suggest that breathing pattern is influenced by both specific afferent input from chemoreceptors and generalised afferent input while chemoreceptors specific for producing changes in breathing pattern may exist in fish.
\end{abstract}

(C) 2003 Elsevier Science B.V. All rights reserved.

Keywords: Control of breathing, breathing pattern, afferent input; Fish, tambaqui (Colossoma macropomum); Gill, fish, denervation; Nerve, vagus, glossopharyngeus; Pattern of breathing, afferent input

\footnotetext{
* Corresponding author. Tel.: +1-416-287-7426; fax: +1416-287-7642.

E-mail address: sgreid@utsc.utoronto.ca (S.G. Reid).

1 Present address: Department of Zoophysiology, University of Göteborg, Box 463, S-405 30 Gothenburg, Sweden.
}

\section{Introduction}

In all vertebrate groups, breathing patterns arise that can be classified as either continuous or periodic (Milsom, 1991). Periodic breathing can be further classified as either 'regular' where the breaths are infrequent but relatively even spaced, 'random' where the breaths occur with no obvious rhythm or episodic where breaths are clustered 
together into episodes separated by discrete nonventilatory periods. These non-ventilatory periods may either be periods of breath holding or of apnea. These breathing patterns originate as the rhythmic neural output from a central respiratory rhythm generator located within the medulla (Richter, 1982) that is conditional in nature, operating at a sub-threshold level that requires some external (afferent) input to initiate breathing (Feldman et al., 1990). Ultimately, the pattern of motor output to the respiratory muscles depends upon modification of the respiratory rhythm by various afferent and central inputs (Ballintijn and Juch, 1984; Feldman et al., 1990). These include inputs from chemoreceptors (e.g. Smatresk, 1990; Burleson et al., 1992), mechanoreceptors (Ballintijn and Bamford, 1975; de Graff and Ballintijn, 1987; de Graff et al., 1987; Milsom, 1990) and higher brain centres (Ballintijn and Juch, 1984; Roberts and Ballintijn, 1988; Smatresk, 1990; Kinkead, 1997).

In water-breathing fish, respiratory-related afferent input to the brain arises from mechanoreceptors on the gill arches and $\mathrm{O}_{2}$ and $\mathrm{CO}_{2} / \mathrm{pH}$ chemoreceptors located on the gill arches and in the oro-branchial cavity (mouth and operculum) (e.g. Randall and Jones, 1973; Smatresk et al., 1986; Burleson and Smatresk, 1990; Reid et al., 2000; Sundin et al., 1999, 2000; McKendry et al., 2001; Milsom et al., 2002). These receptors can monitor gill displacement and either $\mathrm{O}_{2}$ or $\mathrm{CO}_{2} /$ $\mathrm{pH}$ levels in the water or blood and, once stimulated, initiate changes in cardiorespiratory function (see Smatresk, 1990; Burleson et al., 1992; Milsom et al., 1999 for reviews). The mechanoreceptors and chemoreceptors on the gill arches are innervated by branches of the glossopharyngeal (IXth cranial; cnIX) or vagus (Xth cranial; cnX) nerves while the chemoreceptors in the oro-branchial cavity are innervated by branches of the trigeminal (Vth cranial; $\mathrm{cnV}$ ) and facial (VIIth cranial; cnVII) nerves.

Previously we have analysed the effects of stimulation of $\mathrm{O}_{2}$ and $\mathrm{CO}_{2} / \mathrm{pH}$ chemoreceptors in the gills and oro-branchial cavity on the cardiorespiratory responses to hypoxia and hypercarbia in a Neotropical fish, the tambaqui (Sundin et al., 2000; Milsom et al., 2002). Tambaqui are water- breathing, hypoxia-tolerant (p50 $=2.4$ Torr; Brauner et al., 2001) fish that perform aquatic surface respiration (ASR) under conditions of severe environmental hypoxia (Val and Almeida-Val, 1995; Rantin and Kalinin, 1996). Under these conditions, they remain at the surface of the water where they skim the $\mathrm{O}_{2}$-rich surface layer. To facilitate this, the lower lip swells and acts as a funnel that directs the surface water into the mouth and over the gills (Saint-Paul, 1988). During the course of these studies (Sundin et al., 2000; Milsom et al., 2002) we noticed that reduced chemoreceptor drive (i.e. hyperoxia) and/or removal of chemoreceptor input via denervation not only altered total ventilation but, at times, also altered the pattern of breathing in tambaqui. Based on our observations, we hypothesised that input from $\mathrm{O}_{2}$ and $\mathrm{CO}_{2} / \mathrm{pH}$ chemoreceptors in the gills and oro-branchial cavity not only produced respiratory drive affecting total ventilation (Sundin et al., 2000; Milsom et al., 2002), but also produced 'biasing input' altering breathing pattern. The goal of this study, therefore, was to quantify the effects of changing respiratory drive and the inputs from various receptor groups on the breathing pattern of tambaqui. The study addressed the role of both specific and general afferent input in the formation of complex breathing patterns in the tambaqui and explored the possibility that chemoreceptors specific for producing changes in breathing pattern (versus total level of ventilation) may exist in fish.

\section{Materials and methods}

\subsection{Animals}

Juvenile tambaqui (Colossoma macropomum) (500-1000 g) were obtained from CEPTA (Tropical Fish Research Center)/IBAMA in Pirassununga, SP, Brazil and transported to the Federal University of São Carlos. These fish were third- or fourth-generation descendants of native Tambaqui taken from the Amazon in 1993. Animals were maintained outdoors in fibreglass aquaria supplied with aerated, non-chlorinated City of São Carlos tap water. Temperature was maintained at $25^{\circ} \mathrm{C}$, 
and the animals were fed ad libitum every second day. Experiments were performed between January and March.

\subsection{Animal preparation}

Animals were anaesthetised in an aqueous solution of benzocaine $(100 \mathrm{mg} / \mathrm{L})$ pre-dissolved in $2 \mathrm{ml}$ of $70 \%$ ethanol. During surgery the gills were ventilated with a second solution of benzocaine $(50 \mathrm{mg} / \mathrm{ml})$ gassed with air. Impedance electrodes were sutured to each operculum to monitor the breath-by-breath displacement of the operculum and measure ventilation rate and amplitude.

While still anaesthetised, the animals were placed upright in a flow through respirometer such that the gills and operculum were completely submerged in running water while the top of the skull remained above water. Using a Dremmel tool, a hole was drilled through the snout between the nostrils and a conical mask was secured over the snout. A hose for ventilation was placed through the centre of the mask into the mouth of the fish. The animal was ventilated with a solution of the anaesthetic $(50 \mathrm{mg} / \mathrm{ml})$ gassed with air. Using a stereotaxic device and bars fit to the upper edges of the eye sockets, the fish was restrained to secure the head in a constant position. Sponges were placed on either side of the fish to further ensure restraint and prevent the fish from slipping to either side within the box.

Once the fish was restrained, the brain was exposed using a Dremmel tool. The forebrain was removed by suction (decerebration) and the spinal cord severed at the level of the third spinal nerve (similar to the approach of Roberts and Ballintijn, 1988). Once decerebrate and spinalised, anaesthesia was removed and the fish was ventilated with fresh water gassed with air. Spontaneous ventilation began approximately $10 \mathrm{~min}$ following removal of the anaesthetic. Despite continuous artificial (ram) ventilation, the animals always breathed spontaneously. The animals were then allowed to recover for $60 \mathrm{~min}$ prior to the commencement of the experiment.

\subsection{Oro-branchial and central denervation}

\subsubsection{Oro-branchial denervation}

In one group of fish, oro-branchial denervation was performed on the surgery table prior to transfer to the respirometer. To denervate the branches of cranial nerve IX (cnIX) and cranial nerve $\mathrm{X}(\mathrm{cnX})$ to the gill arches, the operculum was reflected forward and a small incision (approximately $1.5 \mathrm{~cm}$ ) was made in the epithelium at the dorsal end of the first and second gill arches where they meet the roof of the opercular cavity. This permitted access to cranial nerve IX (glossopharyngeal) and the branches of cranial nerve $\mathrm{X}$ (vagus) to all gill arches. Tambaqui do not have a pseudobranch. The branchial nerves to all gill arches were dissected free from connective tissue, isolated individually with a fine blunt hook and then severed with fine iris scissors. In this species the nerves lie above the musculature so there is no muscle damage and very little, or no, bleeding during this procedure. The major vagal trunk to the viscera was left intact. Previous experiments have revealed that sham operations, where the nerves were exposed but not cut, had no effect on cardiorespiratory responses to hypoxia and hypercarbia (Sundin et al., 2000).

Branches of the trigeminal $(\mathrm{cnV})$ and facial (cnVII) nerves to the oro-branchial cavity were also sectioned to remove sensory information arising from the mouth and buccal cavity. Two small branches of cnVII were left intact. This was necessary and sufficient to produce opercular movements that could be monitored as an indication of the frequency and amplitude of breathing. The opercular branches of cnVII innervating the floor of the mouth were accessed where they course over the inner surface of the operculum while the palatine branches of cnVII were accessed through a midline incision in the roof of the mouth. The mandibular branches of $\mathrm{cnV}$ innervating the roof of the mouth were accessed bilaterally by rotating the eyes and denervating the nerves, where they coursed over the back of the orbit (Roberts and Ballintijn, 1988), through a small incision in the conjunctiva (see Milsom et al., 2002 for detailed figures). All denervations were confirmed post mortem by autopsy. 


\subsubsection{Central denervation}

In another group of fish, once the animals were decerebrate, spinalised and restrained in the stereotaxic device, the vagus and glossopharyngeal nerves were located at the point of exit from the brain and transected bilaterally with fine iris scissors. These denervations were also confirmed post mortem by autopsy.

\subsection{Experimental protocol}

Three experimental groups were studied: (1) a control group which was decerebrate and spinalised but did not undergo any denervation $(n=12)$, (2) an oro-branchial denervated group in which nerves to the gill arches (cnIX and cnX) and orobranchial cavity ( $\mathrm{cnV}$ and $\mathrm{cnVII}$ ) were cut peripherally ( $n=9$; The branches of $\mathrm{cnX}$ to the lateral line, heart and viscera were still intact in this group) and (3) a central denervated group in which the vagus and glossopharyngeal nerves were cut at the point of exit from the skull $(n=10)$. All branches of $\mathrm{cnX}$ were cut in this later group but $\mathrm{cnV}$ and cnVII remained intact.

Following recovery from anaesthesia, breathing was recorded for $30 \mathrm{~min}$ under normoxic (water gassed with air) conditions. The ventilation water was then rapidly switched to one of four conditions that were created by pre-equilibrating water with the appropriate gas mixture: (1) Hypoxia (water gassed with $\mathrm{N}_{2}$ to achieve a $\mathrm{Pw}_{\mathrm{O}_{2}}=10$ Torr). (2) Hyperoxia (water gassed with pure $\mathrm{O}_{2}$ ). (3) Hypercarbia (water gassed with $5 \% \mathrm{CO}_{2}$ in air). We had determined in a previous study (Sundin et al., 2000) that $5 \% \mathrm{CO}_{2}$ was necessary to produce a reliable respiratory stimulus in this species. With this level of $\mathrm{CO}_{2}$, water $\mathrm{pH}$ fell from approximately 7.0 to 5.0 over a 10 min period. (4) Hyperoxic hypercarbia (water gassed with 5\% $\mathrm{CO}_{2}$ in $\mathrm{O}_{2}$ ). Ventilation was then recorded for 10-15 min for hypoxia and hypercarbia and 20$30 \mathrm{~min}$ for hyperoxia and hyperoxic hypercarbia at which point the animal was once again ventilated with water gassed with air (normoxia). The animal was allowed to recover in normoxic water for $30-$ $60 \mathrm{~min}$ between each experimental condition. The sequence in which fish were exposed to the different gas mixtures was chosen at random.
Water, pre-equilibrated with the different gas mixtures, was re-circulated to ensure constant conditions and limit gas utilisation; temperature was monitored and did not vary from $25^{\circ} \mathrm{C}$.

\subsection{Analytical procedures}

The partial pressure of oxygen $\left(\mathrm{Pw}_{\mathrm{O}_{2}}\right)$ in the water was monitored continuously with an oxygen electrode (FAC 001 oxygen electrode and a FAC 204 oxygen analyser) supplied via siphon, with a steady flow of water from the experimental chamber. The electrode was calibrated with solutions of sodium bisulphate in borax $\left(\mathrm{P}_{\mathrm{O}_{2}}=0\right.$ Torr $)$ and air-equilibrated water $\left(\mathrm{Pw}_{\mathrm{O}_{2}}=140\right.$ Torr at $25^{\circ} \mathrm{C}$ in São Carlos). Water $\mathrm{pH}$ was monitored with a $\mathrm{pH}$ meter. The opercular impedance leads were connected to an impedance converter to measure ventilation and the output was recorded on a Narco chart recorder and a data acquisition system (WINDAQ; DataQ Systems).

\subsection{Data analysis}

Tambaqui exhibited three major breathing patterns. (1) Regular (continuous) breathing which consisted of breathing at a constant frequency and, in most cases, a constant amplitude. (2) Frequency cycling which was a form of continuous breathing where breathing frequency waxed and waned. In some animals (see Section 3) the amplitude of the breaths also changed during these cycles. (3) Episodic breathing where breaths were clustered into discrete episodes separated by periods of no active ventilation. For each experimental condition the breathing pattern was classified based on examination of the entire recording period. In all cases the breathing pattern was easily characterised as either regular, frequency cycling or episodic.

The occurrence of the different breathing patterns was quantified and is reported as the percentage of animals exhibiting a given breathing pattern under a given condition. During frequency cycling, the delineation between fast and slow cycles was, for the most part, obvious. There were, however, some cases (i.e. Fig. 1d) in which an element of subjectivity was required. The values 


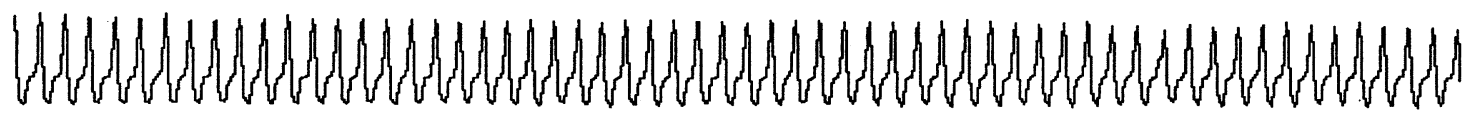 \\ (A) \\ $5 \mathrm{sec}$}
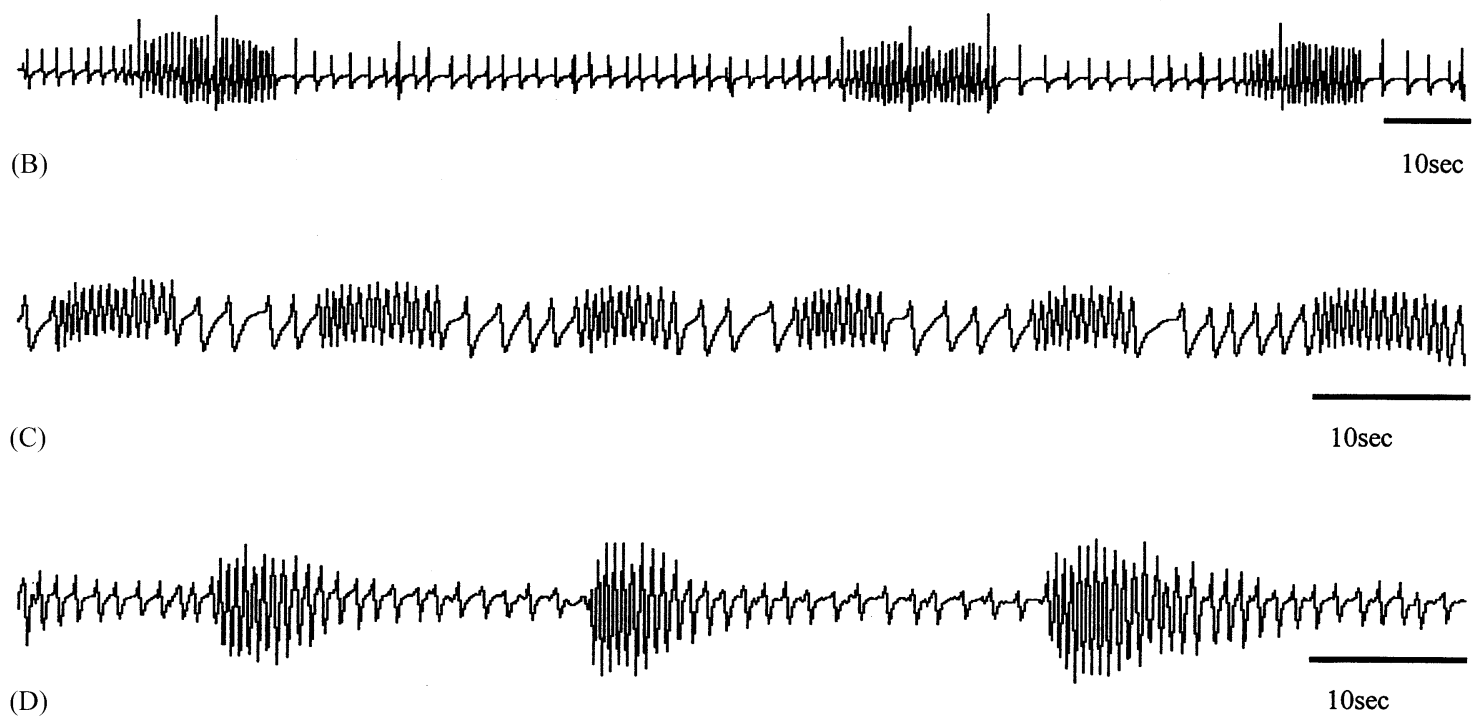

Fig. 1. (A) Continuous breathing and (B-D) examples of frequency cycling in the decerebrate, artificially-ventilated tambaqui. (A) Continuous breathing in intact (decerebrate) tambaqui under normoxic conditions. (B) Frequency cycling following central denervation under normoxic conditions. (C) Frequency cycling in intact animals under hyperoxic conditions. (D) Frequency cycling in intact animals under hyperoxic conditions. In part $\mathrm{D}$, note the progressive decrease in frequency and amplitude as the breathing pattern transforms from high frequency, large amplitude breaths to low frequency, low amplitude breaths.

for breathing frequencies within the fast and slow cycles during frequency cycling are reported as the mean \pm 1 standard error of the mean (S.E.M.). The breathing frequency within the fast and slow cycles was compared with a $t$-test. The limit of significance was taken to be $5 \%$.

Breathing episodes were designated as such when the pause between two successive breaths was no longer than the length of two breathing cycles according to previously described criteria (Kinkead and Milsom, 1997; Reid and Milsom, 1998). The number of fictive breaths within an episode was obtained by counting the number of breaths that occurred in succession with no pause longer than the length of two breathing cycles between them.

\section{Results}

\subsection{Description of the various breathing patterns}

In this study the tambaqui exhibited three major breathing patterns; regular continuous breathing, frequency cycling and episodic breathing. Under some conditions they stopped breathing all together. Apnea could be sustained under these conditions since these animals were artificially ventilated throughout the experiment. Fig. 1a illustrates an example of regular breathing in a control decerebrate tambaqui under normoxic conditions. In this case, breathing occurred continuously with a constant frequency and amplitude. 
We define frequency cycling as continuous breathing during which breathing frequency alternated between rapid periods and slow periods. Fig. $1 b-d$ illustrates three different examples of frequency cycling. In Fig. 1b (following central denervation; normoxia) and Fig. 1c (intact; hyperoxia), the transition from fast to slow breathing, and vice versa, was obvious. However, in Fig. 1d (intact; hyperoxia), the transition from fast to slow breathing occurred gradually and it was harder to delineate where the fast period ended and slow period began. On the other hand, the point of transition from the slow period to the fast period occurred without any ambiguity. Fig. 1c illustrates frequency cycling with relatively constant ventilatory amplitude while in Fig. 1d the rapid breaths also had a greater amplitude than the slower breaths.

Although the delineation between fast and slow periods contained an element of subjectivity, the frequency of breathing within the fast and slow cycles was $1.43 \pm 0.07$ and $0.84 \pm 0.05$ breaths $/ \mathrm{sec}$, respectively. These values were statistically different $(P<0.05)$ and the breathing frequency within these cycles was not affected by the denervation state or the treatment (i.e. hypoxia, hypercarbia, hyperoxia or hyperoxic hypercarbia).

Fig. 2 illustrates three examples of episodic breathing in decerebrate tambaqui. All traces in Fig. 2 were recorded following central denervation under normoxic conditions. Fig. 2a illustrates discrete breathing episodes separated by periods of no active ventilation (apnea). The middle episode from Fig. 2a is illustrated on an expanded scale in Fig. 2b. In this case, the transition from the breathing episode to the period of no ventilation involved the occurrence of three slower breaths reminiscent of the 'run down' observed between fast and slow cycles in Fig. 1d. Fig. 2c illustrates episodic breathing from another fish where the episodes were more frequent that those in Fig. 2a. Fig. 2d illustrates one of the episodes from Fig. 2c on an expanded scale. Fig. 2e illustrates an episodic breathing pattern with two to nine breaths per episode separated by relatively short periods of no breathing.

Fig. 3a (control group; hyperoxia) illustrates a continuous breathing pattern in which the breath amplitude progressively increased and decreased in a cyclical manner. This pattern is shown on an expanded scale in Fig. 3b and resembles human Cheyne-Stokes breathing (see Section 4). Although the breath amplitude became very small, breathing never stopped entirely. Fig. 3c illustrates an episodic breathing pattern in which the breath amplitude is large at the beginning of the episode and progressively decreased throughout the episode until breathing stopped. Fig. 3d illustrates one of these episodes on an expanded scale.

\subsection{Breathing patterns during normoxia}

The percentage of fish exhibiting each breathing pattern under normoxic conditions, in each denervation group, is illustrated in Fig. 4. When the animals were intact (decerebrate and artificially ventilated within the stereotaxic device), 50\% breathed continuously (regular breathing) and $50 \%$ exhibited a pattern of frequency cycling (Fig. 4a). Following oro-branchial denervation (Fig. 4b) there was a shift towards frequency cycling with $80 \%$ showing this pattern and only $20 \%$ breathing regularly (continuously). Following central denervation (Fig. 4c) there was no regular, continuous breathing; $20 \%$ of the fish exhibited frequency cycling and $80 \%$ breathed in episodes.

\subsection{Breathing patterns during hyperoxia}

Fig. 4 also illustrates the percentage of animals displaying the various breathing patterns during hyperoxia. From this figure it is apparent that reducing respiratory drive with hyperoxia promotes frequency cycling and episodic breathing rather than continuous regular breathing. In the intact animals (Fig. 4a; no denervation), 83\% exhibited frequency cycling and $17 \%$ breathed in episodes. No animals demonstrated continuous breathing. Following oro-branchial denervation (Fig. 4b), 20\% breathed continuously, 69\% showed frequency cycling and $11 \%$ exhibited no breathing movements. Central denervation (Fig. 4c) resulted in $60 \%$ episodic breathing, $29 \%$ frequency cycling while $11 \%$ of animals showed no breathing movements and no animals breathed continuously. 


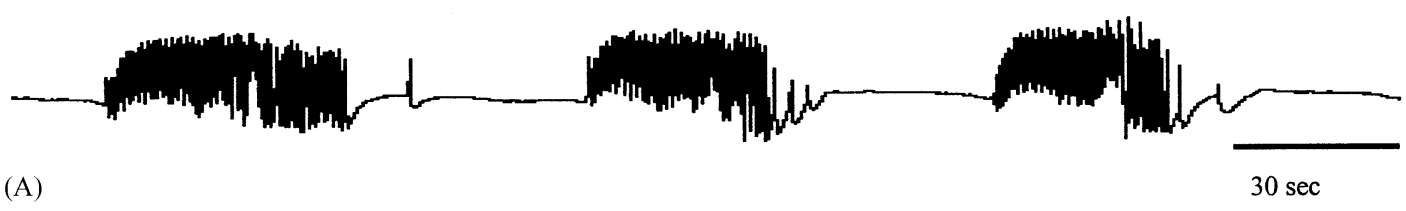

(A)

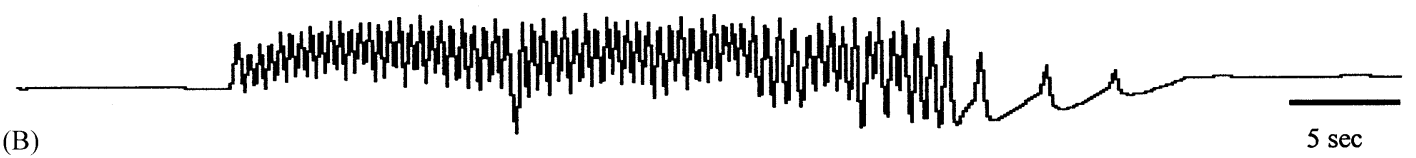

(B)

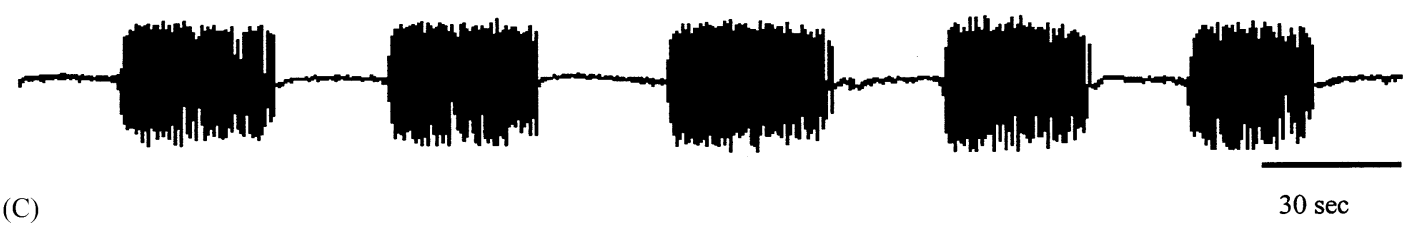

(D)
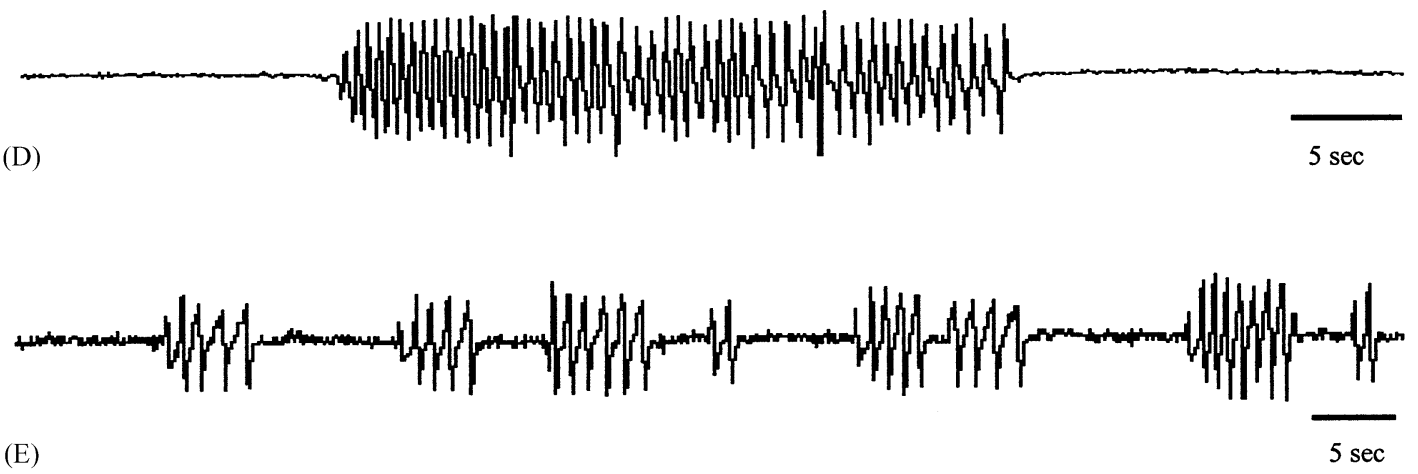

Fig. 2. Examples of episodic breathing in decerebrate, artificially-ventilated tambaqui. (A) Long breathing episodes under normoxic conditions following central denervation. (B) The middle episode from part A shown on an expanded scale. Note the three drawn out breaths that decrease in amplitude following the main component of the episode. (C) Episodic breathing under normoxic conditions following central denervation from a different animal than that illustrated in part A. (D) One of the episodes from part C shown on an expanded scale. (E) A different form of episodic breathing under normoxic conditions following central denervation. Compared with parts $\mathrm{A}$ and $\mathrm{C}$, these episodes are shorter; containing fewer breaths per episode.

\subsection{Breathing patterns during hypoxia}

The breathing patterns exhibited during hypoxia are also illustrated in Fig. 4. In the intact animals (Fig. 4a) $83 \%$ breathed regularly and 17\% showed frequency cycling. No animals breathed in episodes. Surprisingly, despite total denervation of gill arches and oro-branchial cavity (Fig. 4b; orobranchial denervation), there was a shift from the predominant pattern of frequency cycling during normoxia to predominantly continuous breathing
$(67 \%)$ during hypoxia. In the centrally denervated animals (Fig. 4c), hypoxia resulted in the predominance of continuous breathing $(50 \%)$ and frequency cycling $(40 \%)$ compared with the predominant pattern of episodic breathing observed during normoxia.

\subsection{Breathing patterns during hypercarbia}

The breathing patterns exhibited during hypercarbia are illustrated in Fig. 5 and were very 


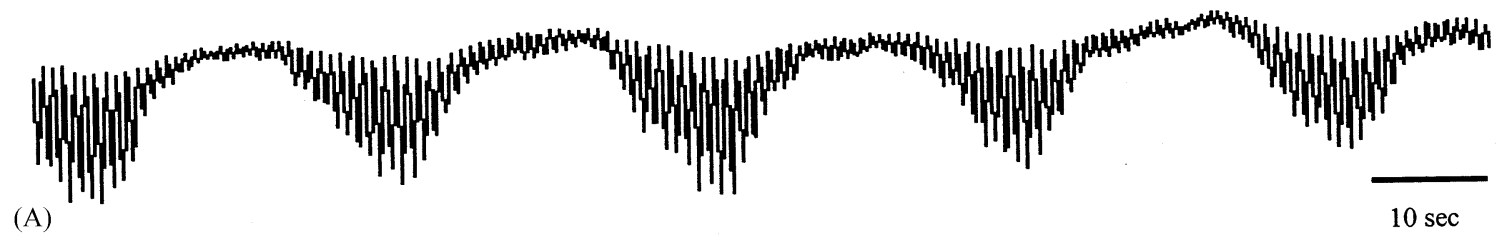

(B)

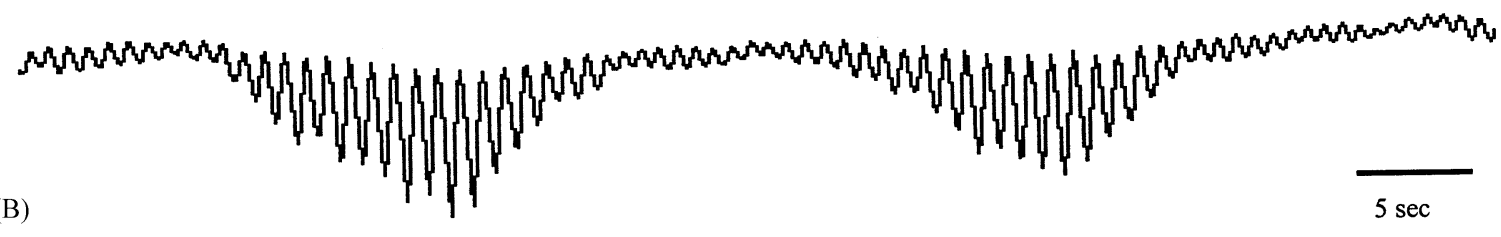

(C)

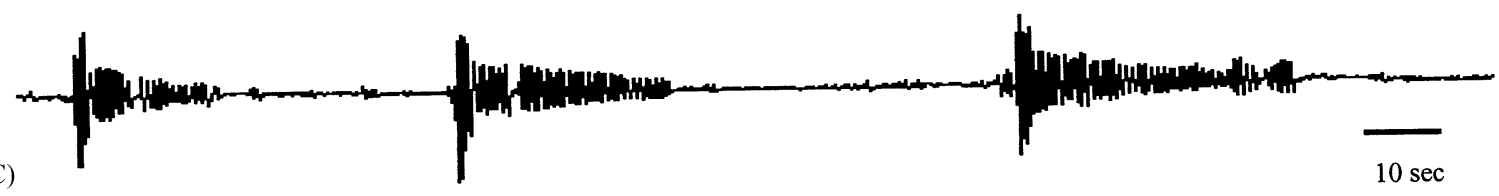

(D)

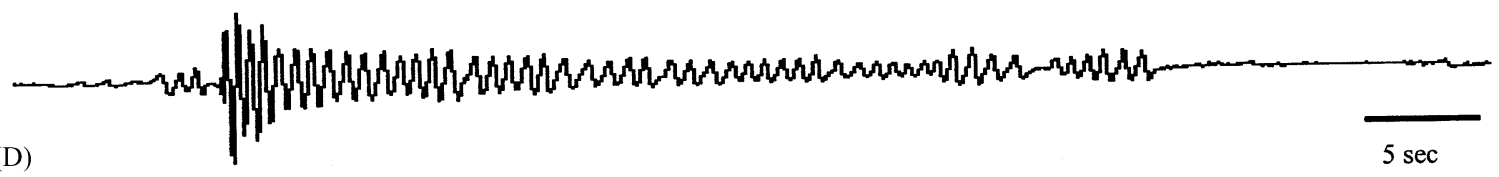

Fig. 3. (A) An example of a breathing pattern (control group; hyperoxia) in which breath amplitude waxes and wanes while frequency remains constant. (B) The trace from part A shown on an expanded scale. (C) An episodic breathing pattern (control group; hyperoxia) in which the breath amplitude decreases progressively throughout the episode until breathing stops. (D) The last episode from part C shown on an expanded scale.

similar to those observed during hypoxia. The data for normoxia (and normocarbia) are re-plotted on this figure for comparison. In the intact animals (Fig. 5a), 83\% breathed regularly and 17\% showed frequency cycling. No animals breathed in episodes. Following total denervation of gill arches and oro-branchial cavity (Fig. 5b; oro-branchial denervation), $88 \%$ breathed continuously and $12 \%$ exhibited frequency cycling. In the centrally denervated animals (Fig. 5c), hypercarbia resulted in $56 \%$ continuous breathing, $33 \%$ frequency cycling with $11 \%$ breathing in episodes.

\subsection{Breathing patterns during hyperoxic hypercarbia}

Fig. 5 also illustrates the various breathing patterns during exposure to hyperoxic hypercarbia. In the intact animals (Fig. 5a) the response was identical to hypercarbia alone with $83 \%$ of the animals breathing regularly and $17 \%$ showing a pattern of frequency cycling. Following oro-branchial denervation (Fig. 5b), $50 \%$ of the animals breathed continuously and 50\% showed frequency cycling. This response lies in between the responses 

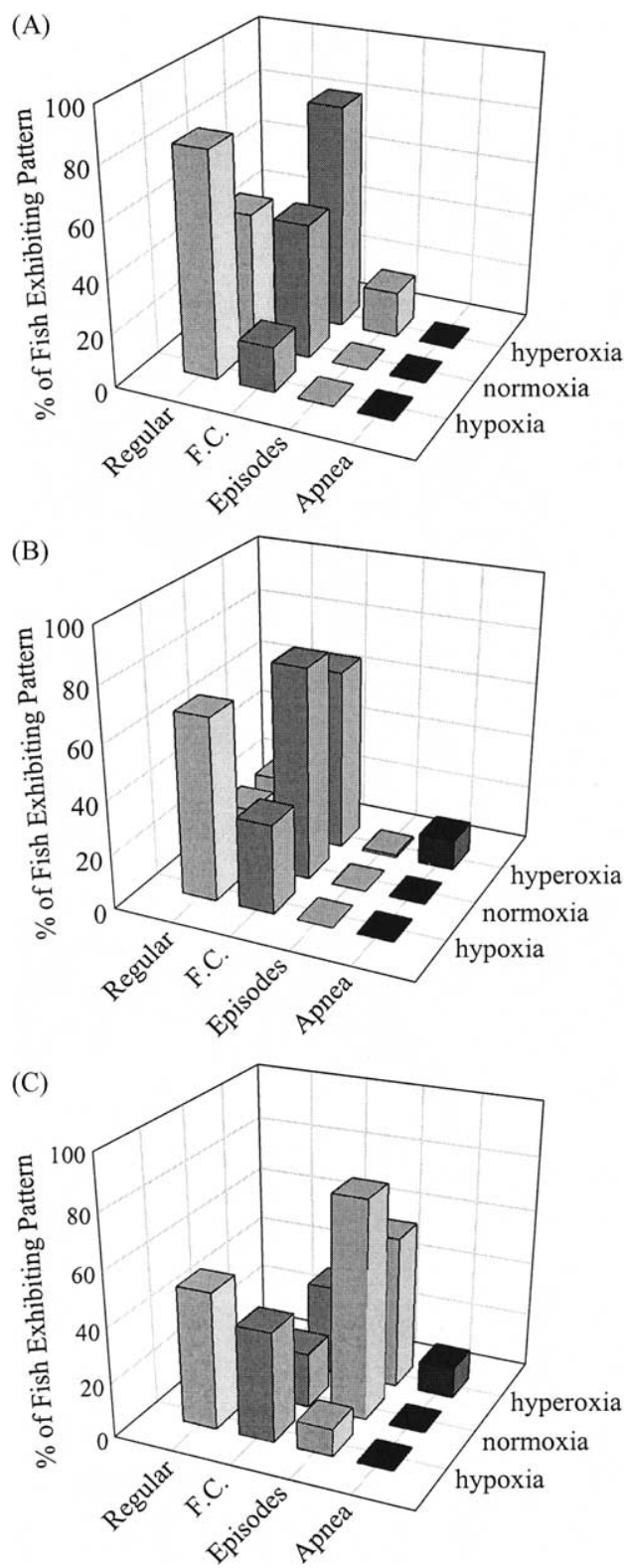

Fig. 4. The percentage of fish displaying a particular breathing pattern (continuous, frequency cycling, episodic breathing or apnea) during normoxia, hypoxia or hyperoxia. (A) The intact (control; decerebrate) group with no denervation. (B) The orobranchial denervation group with all nerves ( $\mathrm{cnIX}$ and $\mathrm{cnX}$ ) to the gill arches and musculature sectioned as well as the sensory branches of $\mathrm{cnV}$ and cnVII to the mouth and operculum. (C) The central denervation group with the vagus and glossopharyngeal nerves transected as they left the skull. Note that orobranchial denervation promoted frequency cycling while central denervation promoted episodic breathing. to hypercarbia and hyperoxia alone. In the centrally denervated animals (Fig. 5c), hyperoxic hypercarbia resulted in $30 \%$ of animals breathing regularly and $70 \%$ exhibiting frequency cycling. As such it appears as if the response to hyperoxic hypercarbia (in the centrally denervated animals) is more like hypercarbia than hyperoxia. Interestingly, in the centrally denervated animals no episodic breathing was seen during hyperoxic hypercarbia.

\section{Discussion}

\subsection{Water and blood gases}

The levels of hypoxia $\left(\mathrm{Pw}_{\mathrm{O}_{2}}=10\right.$ Torr $)$ and hypercarbia (water gassed with $5 \% \mathrm{CO}_{2}$ ) used in this study were chosen based on previous work in which tambaqui exhibited a robust ventilatory response to these treatments (Sundin et al., 2000; Milsom et al., 2002). Given the low p50 value in tambaqui (2.4 Torr; Brauner et al., 2001), a water $\mathrm{P}_{\mathrm{O}_{2}}$ of 10 Torr is unlikely to be extreme for this species. Recently, Rantin and co-workers exposed tambaqui to $5 \% \mathrm{CO}_{2}$-equilibrated water for $6 \mathrm{~h}$ (Florindo et al., unpublished data). In those experiments, breathing steadily increased during the first hour of hypercarbia and then remained constant, at the elevated level, for the remainder of the $6 \mathrm{~h}$ period. When normocarbia was restored, breathing quickly returned to resting levels. Given that tambaqui tolerate this level of hypercarbia for $6 \mathrm{~h}$, it is reasonable to assume that no detrimental effects were occurring over the 10-15 min time period in this current study. Furthermore, based on our previous pilot experiments, tambaqui do not exhibit a ventilatory response during short term exposure to water equilibrated with 1.25 or $2.5 \% \mathrm{CO}_{2}$; hence the decision to use $5 \% \mathrm{CO}_{2}$ equilibrated water which produces a robust hypercarbic ventilatory response allowing for the study of respiratory chemoreflexes and breathing pattern formation.

Although blood gas levels were not measured in this study, we have recently measured arterial blood gases with an extracorporeal blood loop in tambaqui during short-term exposure to water 

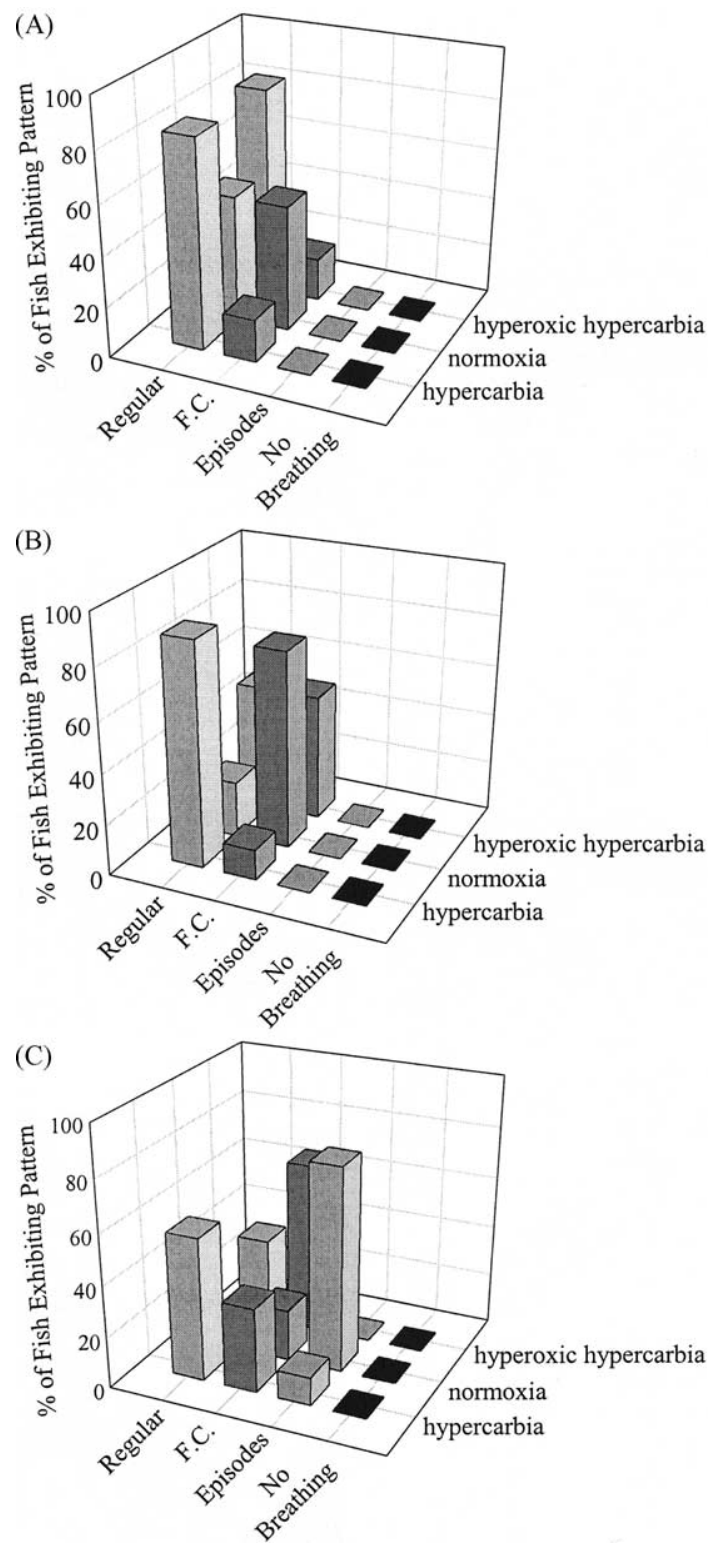

Fig. 5. The percentage of fish displaying a particular breathing pattern (continuous, frequency cycling, episodic breathing or apnea) during normoxia, hypercarbia or hyperoxic hypercarbia. (A) The intact (control; decerebrate) fish with no denervation. (B) The oro-branchial denervation group with all of the nerves (cnIX and $\mathrm{cnX}$ ) to the gill arches and musculature sectioned as well as the sensory branches of $\mathrm{cnV}$ and $\mathrm{cnVII}$ to the mouth and operculum. (C) The central denervation group with the vagus and glossopharyngeal nerves transected as they left the skull. gassed with 5\% $\mathrm{CO}_{2}$ (Perry, Reid, Gilmour, Milsom and Rantin; unpublished). Under these conditions, water $\mathrm{P}_{\mathrm{CO}_{2}}$ increased from approximately 2 to 26 Torr while arterial $\mathrm{P}_{\mathrm{CO}_{2}}$ increased from approximately 3 to 30 Torr and arterial $\mathrm{pH}$ fell from 8.00 to 7.60 . Further elevation of arterial $\mathrm{P}_{\mathrm{CO}_{2}}$ with acetazolamide pre-treatment did not blunt the ventilatory response to hypercarbia. It is clear that short-term exposure of tambaqui to this level of hypercarbia does not have an adverse effect on ventilation. Furthermore, Wood et al. (1998) exposed tambaqui to low levels of water $\mathrm{pH}$ between 6.5 and 3.0. These authors report that plasma glucose, cortisol and total ammonia levels remain constant until water $\mathrm{pH}$ reaches 3.0 indicating a high degree of tolerance to acidic conditions. Wilson et al. (1999) also exposed tambaqui to water $\mathrm{pH}$ levels as low as 3.0 and demonstrated a remarkable insensitivity of the ionoregulatory system of tambaqui to low $\mathrm{pH}$.

\subsection{Breathing patterns in tambaqui}

Three general breathing patterns were observed in these studies: (1) regular continuous breathing; (2) frequency cycling (i.e. continuous breathing with alternating periods of fast and slow breathing with amplitude cycling observed in some instances), and (3) true episodic breathing with periods of ventilation separated by periods of apnea. We feel that it is likely that these breathing patterns represent a continuum from continuous breathing to episodic breathing with frequency cycling occupying an intermediate stage. It would also appear that the continuum extends from episodic breathing to total apnea and that the position of the breathing pattern on this continuum is determined, in part, by the level of respiratory drive.

Regular continuous breathing with a constant frequency and amplitude represents the predominant breathing pattern observed in our previous study on intact (non-decerebrate; non-denervate) tambaqui under normoxic normocapnic conditions (Sundin et al., 2000). In that study, only continuous breathing was observed in $64 \%$ of all animals while frequency cycling was observed in approximately $36 \%$ of animals. These values were 
obtained from the original data traces from that study (Sundin et al., 2000). The decerebrate, stereotaxically mounted preparation used in the present study exhibited a similar breathing pattern distribution under normoxic conditions suggesting that decerebration and immobilisation did not alter the normal breathing pattern distribution. Decerebrate dogfish also breathed in a normal manner (Roberts and Ballintijn, 1988).

Frequency cycling or waxing and waning of breathing frequency, and sometimes amplitude, is not uncommon in fish. Roberts and Ballintijn (1988) reported that breathing rhythm in a stereotaxically-held decerebrate dogfish would also shift frequency to faster or slower rates. As was the case in the current study, these authors also observed that breathing amplitude often changed such that the periods of fast breathing were associated with larger amplitude breaths.

Given the low levels of $\mathrm{O}_{2}$ in water, most fish tend to breathe continuously. However, some water-breathing fish can breathe episodically under conditions of low metabolic rate in welloxygenated water (Ballintijn, 1988; Juch and Ballintijn, 1983; Juch and Luiten, 1981; Lomholt and Johansen, 1979). Additionally, all air-breathing fish breathe air periodically in order to obtain oxygen (see Smatresk, 1989 for review) while ventilating their gills continuously to facilitate some $\mathrm{CO}_{2}$ excretion and ionic/osmotic regulation (see Randall, 1990 for review).

\subsection{Role of chemoreceptor inputs in breathing pattern formation}

The position of the breathing pattern on the continuum appears to be determined, at least in part, by the level of respiratory drive. Based on the predominance of frequency cycling during both normoxia following oro-branchial denervation and hyperoxia in the intact state, it is likely that one resting drive to breathe that biases the position of the breathing pattern on the continuum originates from either gill and/or oro-branchial chemoreceptor input. Sundin et al. (2000) reported little change in resting levels of ventilation frequency following progressive branchial denervation (cnIX and $\mathrm{cnX}$ ) suggesting that the resting drive that modulates pattern likely arises from chemoreceptors innervated by $\mathrm{cnV}$ or cnVII.

Previous studies (Sundin et al., 2000; Milsom et al., 2002) have identified the location and innervation of the chemoreceptors involved in controlling ventilation rate and amplitude during hypoxia and hypercarbia in tambaqui. Central denervation of cnIX and cnX did not abolish the increase in ventilation amplitude observed during hypoxia in decerebrate tambaqui (Milsom et al., 2002) nor did it abolish the shift towards continuous breathing in the present study. This suggests that some of the changes in ventilation and breathing pattern seen during hypoxia arise from receptors that are innervated by nerves other than cnIX and cnX. The same was true for the changes seen during hypercarbia.

In our previous study, oro-branchial denervation (cnV, cnVII, cnIX and cnX) eliminated all cardiorespiratory reflexes to hypoxia and hypercarbia (Milsom et al., 2002). In the current study, oro-branchial denervation did not eliminate the shift in the breathing pattern continuum from predominately frequency cycling (normoxia, post oro-branchial denervation) to predominately continuous breathing (hypoxia, post oro-branchial denervation). This suggests that changes in breathing pattern during hypoxia may be partially regulated by $\mathrm{O}_{2}$ chemoreceptors not denervated in this study. As a consequence, we know of no chemoreceptor loci that can explain the changes in breathing pattern observed with changes in respiratory drive following oro-branchial denervation in our decerebrate tambaqui. The two branches of cnVII that were left intact innervate opercular muscle. Given this, and the results of Milsom et al. (2002), it is unlikely that these two nerves innervate chemoreceptor loci that are responsible for these changes in breathing pattern. We cannot, however, completely exclude this possibility. A similar situation occurred during exposure to hypercarbia and hyperoxic hypercarbia following oro-branchial denervation. These observations raise two important questions: (1) where are the chemoreceptors involved in changing breathing pattern during changes in respiratory drive? (2) Are there chemoreceptors that can trigger changes in breathing pattern but do not 
mediate changes in breathing frequency or amplitude?

It is possible that changes in breathing pattern may be induced by stimulation of central chemoreceptors although there is as yet no evidence that water breathing fish possess central chemoreceptors for either $\mathrm{CO}_{2} / \mathrm{pH}$ or $\mathrm{O}_{2}$. Wilson et al. (2000) and Sanchez et al. (2001) however have recently reported the presence of central $\mathrm{pH} / \mathrm{CO}_{2}$ chemosensitivity in the gar and South American lungfish (both air-breathers), respectively. Our own attempts to alter breathing in the tambaqui by superfusing the dorsal surface of the brain with hypoxic, hypercapnic, hyperoxic, acidic and alkaline solutions were without effect (Milsom et al., 2002). In this regard our results are consistent with those of other investigators who have failed to demonstrate the presence of central chemoreceptors in water-breathing fish (Rovainen, 1977; Kawasaki, 1980; Hedrick et al., 1991). Currently the location of the chemoreceptors involved in changing breathing pattern, with changes in respiratory drive following oro-branchial denervation, is unknown.

\subsection{Role of mechanoreceptor and non-specific inputs in breathing pattern formation}

It is possible that changes in breathing pattern following gill denervation also resulted, in part, from removal of mechanoreceptor feedback. Working with isolated dogfish gills and recording from afferent nerve fibres, Satchell and Way (1962) observed that a population of gill proprioceptors increased their discharge rate when a gill process (raker) was deflected and again when it returned to the neutral position. These phasic changes also occurred superimposed on a tonic increase in firing caused by a maintained deflection of the gill process. These authors suggested that gill proprioceptors were ideally suited to report both the pharyngeal movements associated with respiration and any maintained displacement of the operculum that may occur during swimming. Satchell (1961) observed that in partially curarised dogfish, in which the opercular flaps could still ventilate the gills sufficiently to record breathing, increases in ventilation amplitude were not asso- ciated with the usual decrease in breathing frequency. Based on these two studies, Satchell and Way (1962) suggested that feedback from gill proprioceptors is involved in matching breathing frequency to the amplitude of respiratory movements. They suggested that proprioceptors modulate the time taken for 'inspiration' (abduction) and the pause before 'expiration' (adduction) in proportion to the amplitude of the previous breath. In this manner, if amplitude increased, rate would decrease, and vice versa, to maintain regular breathing and minute ventilation. Presumably the simultaneous increase in both breathing amplitude and rate, observed during hypoxia and hypercarbia in most fish species, results from chemoreceptor input overriding any proprioceptor input that would tend to decrease one variable if the other increased.

Roberts and Ballintijn (1988), working on paralysed dogfish, demonstrated that vagal sensory activity was important in maintaining the stability of the central respiratory circuits. These authors suggest that sensory vagal feedback, originating from gill mechanoreceptors, serves to facilitate an efficient change in respiratory rhythm under appropriate conditions and to prevent inappropriate rhythm changes. In the current study, the removal of peripheral input from both mechanoreceptors and chemoreceptors, following oro-branchial denervation, may have prevented the occurrence of regular constant breathing under normoxic conditions; resulting in frequency cycling being the predominant form of breathing.

Ballintijn et al. (1983) demonstrated that vagal stimulation in the carp modified breathing pattern by changing the timing of the individual breath in a manner dependent upon the time in the breath when the stimulus was presented. The breath was always shortened when stimuli were given during the abduction phase; abduction was terminated and adduction increased in speed. Only the ongoing breath was modified and subsequent breaths were unchanged. That vagal stimulation only affected the ongoing breath and not subsequent breaths, however, suggests that mechanisms involved in the clustering of breaths together into episodes do not arise exclusively from the gills. This is consistent with the idea advanced by 
Milsom et al. (1997) that episodic breathing in most vertebrates is produced by the central nervous system in the absence of peripheral input. In the current study, the emergence of episodic breathing following central denervation of cnIX and cnX suggests that some feedback or input from cnIX or $\mathrm{cnX}$ prevents episodic breathing under most conditions and that episodes are only well developed following central denervation of cnIX and cnX. Sundin et al. (2000) did not observe periodic breathing following peripheral denervation of cnIX and cnX to the gills. As such, it appears as if afferent input from cnIX and cnX, originating from a site other than the gills, is influencing the emergence of periodic breathing.

\subsection{Breathing pattern following oro-branchial denervation versus central denervation}

Central denervation of cnIX and cnX produced different effects on the breathing pattern than did oro-branchial denervation. Following central denervation, breathing was predominantly episodic during normoxia and hyperoxia as opposed to the predominance of frequency cycling following orobranchial denervation. These results suggest that both specific afferent input from sources such as gill and oro-branchial chemoreceptors or mechanoreceptors and other (primarily non-respiratoryrelated) afferent input from a complex nerve such as the vagus may both function to modify the breathing pattern continuum independently of one another. As such, both a reduction in the drive to breathe (as illustrated by hyperoxia and orobranchial denervation) and the removal of afferent input from a site other than the gills and orobranchial cavity (as illustrated by the differences between oro-branchial and central denervation) may contribute independently to breathing pattern formation in the tambaqui.

\subsection{Summary and perspectives}

The results of this study indicate that: (1) the breathing pattern in the tambaqui forms a continuum and ranges from regular continuous breathing to frequency cycling and to episodic breathing. Various forms of afferent input can modify and shape these complex breathing patterns. (2) Removal of specific afferent input from peripheral chemoreceptors by oro-branchial denervation or hyperoxia facilitates the expression of frequency cycling. (3) A more generalised removal of afferent input via central denervation of cnIX and $\mathrm{cnX}$ promotes an episodic breathing pattern. (4) Chemoreceptors specific for producing changes in breathing pattern (versus total level of ventilation) may exist in fish.

In lower vertebrates such as amphibians, episodic breathing results from interactions between central processes and afferent input from peripheral chemoreceptors and mechanoreceptors (Kinkead, 1997; Milsom et al., 1997). However, it is becoming increasingly clear that, in these animals, the central nervous system can produce episodes in the absence of any peripheral input. In the tambaqui, it is possible to envision a scenario in which central processes produce breathing episodes or fast cycles. On the other hand, peripheral feedback may determine whether the fast breathing will be followed by apnea (periodic breathing) or a slow cycle (frequency cycling). If respiratory drive is low, apnea will follow the fast breathing and result in periodic breathing. If drive is higher, a slow cycle will follow the fast cycle and frequency cycling will occur. At very high levels of respiratory drive (i.e. during hypoxia or hyperoxia), enhanced levels of peripheral feedback could counter the tendency of the central nervous system to produce episodes; resulting in continuous breathing.

Frequency/amplitude cycling and episodic breathing described here are very similar to Cheyne-Stokes breathing with and without apnea. During Cheyne-Stokes breathing there are cycles of gradual increases in tidal volume followed by a gradual decrease in tidal volume and a period of apnea. This pattern of breathing is seen in humans with CNS disease or medullary lesions, in congestive heart failure patients and in otherwise healthy people during sleep at high altitudes. In this later case, Cheyne-Stokes breathing occurs due to increased feedback from $\mathrm{O}_{2}$ chemoreceptors in conjunction with decreased feedback from $\mathrm{pH} /$ $\mathrm{CO}_{2}$ chemoreceptors. Reduced levels of inspired $\mathrm{O}_{2}$ lead to increases in breathing, which, in turn, 
lowers arterial $\mathrm{P}_{\mathrm{CO}_{2}}$, leading to apnea. During apnea, arterial $\mathrm{P}_{\mathrm{O}_{2}}$ decreases and $\mathrm{P}_{\mathrm{CO}_{2}}$ increases leading to the occurrence of a breathing episode. The cycle then repeats itself. In other words, changes in respiratory-related peripheral feedback alter the breathing pattern in addition to overall levels of ventilation. The observation that both fish and humans express similar breathing patterns, other than regular continuous breathing, suggests that the neural control mechanisms involved in respiratory control are similar amongst vertebrates.

\section{Acknowledgements}

This study was supported by Natural Sciences and Engineering Research Council (NSERC) of Canada scientific and operating grants to W.K. Milsom and FAPESP (Fundação de Amparo à Pesquisa do Estado de São Paulo) and CNPq (the Brasilian National Research Council for Development of Sciences and Technology) grants to F.T. Rantin. L. Sundin was the recipient of Swedish Foundation for International Cooperation in Research and Higher Education (STINT) and Isaak Walton Killam Foundation postdoctoral fellowships. Travel grants for L. Sundin were provided by the Wenner-Gren-Centre Foundation and the Royal Society of Arts and Sciences in Göteborg. S.G. Reid is currently supported by a Parker B. Francis Fellowship from the Francis Family Foundation.

\section{References}

Ballintijn, C.M., 1988. Evolution of central nervous control of ventilation in vertebrates. In: Taylor, E.W. (Ed.), The Neurobiology of the Cardio-Respiratory System. Manchester University Press, Manchester, pp. 3-27.

Ballintijn, C.M., Bamford, O.S., 1975. Proprioceptor motor control in fish respiration. J. Exp. Biol. 62 (1), 99-114.

Ballintijn, C.M., Juch, P.J.W., 1984. Interaction of respiration with coughing, feeding, vision and oculomotor control in fish. Brain Behav. Evol. 25, 99-108.

Ballintijn, C.M., Roberts, B.L., Luiten, P.G.M., 1983. Respiratory responses to stimulation of branchial vagus nerve ganglia of a teleost fish. Respir. Physiol. 51, 241-257.
Brauner, C.J., Wang, T., Val, A.L., Jensen, F.B., 2001. Nonlinear release of Bohr protons with haemoglobin-oxygenation in the blood of two teleost fishes; carp (Cyprinus carpio) and tambaqui (Colossoma macropomum). Fish Physiol. Biochem. 24, 97-104.

Burleson, M.L., Smatresk, N.J., 1990. Effects of sectioning cranial nerves IX and X on cardiovascular and ventilatory reflex responses to hypoxia and $\mathrm{NaCN}$ in channel catfish. $\mathrm{J}$. Exp. Biol. 154, 407-420.

Burleson, M.L., Smatresk, N.J., Milsom, W.K., 1992. Afferent inputs associated with cardioventilatory control in fish. In: Randall, D.J., Farrell, A.P. (Eds.), Fish Physiology, vol. XIIB. Academic Press, New York, pp. 389-426.

De Graff, P.J., Ballintijn, C.M., 1987. Mechanoreceptor activity in the gills of the carp. II. Gill arch proprioceptors. Respir. Physiol. 69 (2), 183-194.

De Graff, P.J., Ballintijn, C.M., Maes, F.W., 1987. Mechanoreceptor activity in the gills of the carp. I. Gill filament and gill raker mechanoreceptors. Respir. Physiol. 69 (2), $173-$ 182.

Feldman, J.L., Smith, J.C., Ellenberger, H.H., Connelly, C.A., Lui, G.S., Greer, J.G., Lindsay, A.D., Otto, M.R., 1990. Neurogenesis of respiratory rhythm and pattern: emerging concepts. Am. J. Physiol. 259 (5 pt 2), R879-R886.

Hedrick, M.S., Burleson, M.L., Jones, D.R., Milsom, W.K., 1991. An examination of central chemosensitivity in an air-breathing fish (Amia calva). J. Exp. Biol. 155, 165174.

Juch, P.J.W., Ballintijn, C.M., 1983. Tegmental neurons controlling medullary respiratory centre activity in the carp. Respir. Physiol. 51, 95-107.

Juch, P.J.W., Luiten, P.G.M., 1981. Anatomy of respiratory rhythmic systems in brainstem and cerebellum. Brain Res. 230, 51-64.

Kawasaki, R., 1980. Maintenance of respiratory rhythm generation by vascular perfusion with physiological saline in the isolated head of the carp. Jpn. J. Physiol. 30, 575589.

Kinkead, R., 1997. Episodic breathing in frogs. Converging hypotheses on neural control of respiration in air-breathing vertebrates. Am. Zool. 37 (1), 31-40.

Kinkead, R., Milsom, W.K., 1997. Role of pulmonary stretch receptor feedback in control of episodic breathing in the bullfrog. Am. J. Physiol. 272, R497-R508.

Lomholt, J.P., Johansen, K., 1979. Hypoxia acclimation in carp-how it effects $\mathrm{O}_{2}$ uptake, ventilation, and $\mathrm{O}_{2}$ extraction from water. Physiol. Zool. 52, 38-49.

McKendry, J.E., Milsom, W.K., Perry, S.F., 2001. Branchial $\mathrm{CO}_{2}$ receptors and cardiorespiratory adjustments during hypercarbia in Pacific spiny dogfish (Squalus acanthias). J. Exp. Biol. 204 (8), 1519-1527.

Milsom, W.K., 1990. Mechanoreceptor modulation of endogenous respiratory rhythms in vertebrates. Am. J. Physiol. 259 (5 pt. 2), R898-R910.

Milsom, W.K., 1991. Intermittent breathing in vertebrates. Ann. Rev. Physiol. 53, 87-105. 
Milsom, W.K., Harris, M.B., Reid, S.G., 1997. Do descending influences alternate to produce episodic breathing. Respir. Physiol. 110 (2-3), 307-317.

Milsom, W.K., Sundin, L., Reid, S.G., Kalinin, A.L., Rantin, F.T., 1999. Chemoreceptor control of cardiovascular reflexes. In: Val, A.L., Almeida-Val, V.M.F. (Eds.), Biology of Tropical Fishes (Chapter 29). INPA, Manaus, Brazil, pp. $363-374$.

Milsom, W.K., Reid, S.G., Rantin, F.T., Sundin, L., 2002. Extrabranchial chemoreceptors involved in respiratory reflexes in the neotropical fish; Colossoma macropomum (The Tambaqui). J. Exp. Biol. 205, 1765-1774.

Randall, D.J., 1990. Control and coordination of gas exchange in water breathers. In: Advances in Comparative and Environmental Physiology, vol. 6. Springer, Berlin, pp. 253-378.

Randall, D.J., Jones, D.R., 1973. The effects of deafferenation of the pseudobranch on the respiratory response to hypoxia and hyperoxia in the trout (Salmo gairdneri). Respir. Physiol. 17, 291-302.

Rantin, F.T., Kalinin, A.L., 1996. Cardiorespiratory function and aquatic surface respiration in Colossoma macropomum exposed to graded and acute hypoxia. In: Val, A.L., de Almeida-Val, V.M.F., Randall, D.J. (Eds.), Physiology and Biochemistry of the Fishes of the Amazon. INPA, Manaus, pp. 169-180.

Reid, S.G., Milsom, W.K., 1998. Respiratory pattern formation in the isolated bullfrog (Rana catesbeiana) brainstem-spinal cord. Respir. Physiol. 114, 239-255.

Reid, S.G., Sundin, L., Kalinin, A.L., Rantin, F.T., Milsom, W.K., 2000. Cardiovascular and respiratory reflexes in the tropical fish, traira (Hoplias malabaricus): $\mathrm{CO}_{2} / \mathrm{pH}$ chemoresponses. Respir. Physiol. 120, 47-59.

Richter, D.E., 1982. Generation and maintenance of the respiratory rhythm. J. Exp. Biol. 100, 93-107.

Roberts, B.L., Ballintijn, C.M., 1988. Sensory interaction with central generators during respiration in the dogfish. J. Comp. Physiol. A 162 (5), 695-704.

Rovainen, C.M., 1977. Neural control of ventilation in the lamprey. Fed. Proc. 36, 2386-2389.

Saint-Paul, U., 1988. Dinural $\mathrm{O}_{2}$ consumption at different $\mathrm{O}_{2}$ concentrations by Colossoma macropomum and Colossoma brachypomum (Teleostei, Serrasalmidae). Comp. Biochem. Physiol. 89 (A), 675-682.

Sanchez, A.P., Hoffmann, A., Rantin, F.T., Glass, M.L., 2001. Relationship between cerebro-spinal fluid $\mathrm{pH}$ and pulmon- ary ventilation of the South American lungfish, Lepidosiren paradoxa (Fitz.). J. Exp. Zool. 1290 (4), 421-425.

Satchell, G.H., 1961. The response of the dogfish to anoxia. J. Exp. Biol. 38, 531-543.

Satchell, G.H., Way, H.K., 1962. Pharyngeal proprioceptors in the dogfish Squalus acanthias L. J. Exp. Biol. 39, 243-250.

Smatresk, N.J., 1989. Chemoreflex control of respiration in an air-breathing fish. In: Lahiri, S., Forster, R.E., Davies, R., Pack, A.I. (Eds.), Chemoreceptors and Chemoreflexes in Breathing - Cellular and Molecular Aspects. Oxford University Press, London, pp. 52-59.

Smatresk, N.J., 1990. Chemoreceptor modulation of endogenous respiratory rhythm in vertebrates. Am. J. Physiol. 259, R887-R897.

Smatresk, N.J., Burleson, M.L., Azizi, S.Q., 1986. Chemoreflexive responses to hypoxia and $\mathrm{NaCN}$ in longnose gar: evidence for two chemoreceptive loci. Am. J. Physiol. 251, R116-R125.

Sundin, L., Reid, S.G., Kalinin, A.L., Rantin, F.T., Milsom, W.K., 1999. Cardiovascular and respiratory reflexes in the tropical fish, traira (Hoplias malabaricus): $\mathrm{O}_{2}$ chemoresponses. Respir. Physiol. 116, 181-199.

Sundin, L., Reid, S.G., Rantin, F.T., Milsom, W.K., 2000. Branchial receptors and cardiovascular reflexes in a neotropical fish, the tambaqui (Colossoma macropomum). J. Exp. Biol. 203, 1225-1239.

Val, A.L., Almeida-Val, V.M.F., 1995. In: Bradshaw, S.D., Burggren, W., Heller, H.C., Ishii, S., Langer, H., Neuweiler, G., Randall, D.J. (Eds.), Fishes of the Amazon and their Environment: Physiological and Biochemical Aspects. Zoophysiology, vol. 32. Springer, Berlin, Heidelberg, New York.

Wilson, R.W., Wood, C.M., Gonzalez, R.J., Patrick, M.L., Bergman, H.L., Narahara, A., Val, A., 1999. Ion and acidbase balance in three species of Amazonian fish during gradual acidification of extremely soft water. Physiol. Biochem. Zool. 72 (3), 277-285.

Wilson, R.J.A., Harris, M.B., Remmers, J.E., Perry, S.F., 2000. Evolution of air-breathing and central $\mathrm{CO}_{2} / \mathrm{pH}$ respiratory chemosensitivity: new insights from an old fish. J. Exp. Biol. 203, 3505-3512.

Wood, C.M., Wilson, R.W., Gonzalez, R.J., Patrick, M.L., Bergman, H.L., Narahara, A., Val, A.L., 1998. Responses of an Amazonian teleost, the tambaqui (Colossoma macropomum ) to low $\mathrm{pH}$ in extremely soft water. Physiol. Zool. 71 (6), 658-670. 